UVX 2010 (2011) 139-144

DOI: $10.1051 / \mathrm{uvx} / 2011019$

(C) Owned by the authors, published by EDP Sciences, 2011

\title{
Lignes de lumière laser XUV pour les applications scientifiques et technologiques
}

\author{
D. Ros $^{1,2}$, S. Kazamias ${ }^{1,2}$, O. Guilbaud ${ }^{1,2}$, K. Cassou $^{1,2}$, S. Daboussi ${ }^{1,2}$, \\ J. Demailly ${ }^{2}$, M. Pittman ${ }^{1}$, O. Neveu ${ }^{1,2}$, J.-C. Lagron², B. Cros ${ }^{1,2}$ \\ et G. Maynard ${ }^{2}$
}

${ }^{1}$ LASERIX, CLUPS-LUMAT, CNRS-Université Paris-Sud, France

${ }^{2}$ LPGP, CNRS-Université Paris-Sud/Orsay, France

\begin{abstract}
Résumé. LASERIX is a french high-repetition rate laser facility devoted to the realisation of performed XUV laser sources and their use for applications. In this paper we describe the present status and the perspectives of use of LASERIX in the high-repetition rate configuration and the high-Energy one.
\end{abstract}

\section{INTRODUCTION : POURQUOI LASERIX ?}

L'inscription du financement de LASERIX, au Contrat de Plan Etat-Région, pour un montant de 4,2M€, a permis de construire entre 2002 et 2006 une installation laser dédiée aux développements des lasers XUV et leur utilisation pour des expériences d'applications. La naissance de LASERIX était le résultat de nombreux travaux de l'équipe "Lasers XUV et applications" de l'Université Paris-Sud (Orsay) [1-5]. Les progrès réalisés sur les lasers de pompe de courte durée (domaine de la femtoseconde) et les techniques d'amplification associées (Chirped pulse amplification, CPA) ont permis d'aboutir à la fin du siècle dernier à la réalisation de lasers XUV autour de la dizaine de nanomètres (la centaine d'eV) pour des énergies de pompe de quelques dizaines de joules.

Les plasmas chauds et denses dans lesquels peuvent être réalisées des émissions lasers XUV sont produits par une impulsion longue (la centaine de ps) qui crée le plasma et une impulsion courte (la ps) qui permet le chauffage des électrons libres du plasma, qui vont produire les inversions de population par excitation collisionnelle dans les ions lasants (nickeloïdes ou néonoïdes, par exemple).

Depuis lors, des nouveaux travaux ont encore permis de progresser dans l'optimisation du pompage, comme nous le verrons au paragraphe suivant, permettant de réaliser des lasers XUV avec une énergie de l'ordre du Joule et à un taux de répétition de $10 \mathrm{~Hz}$.

L'architecture globale de LASERIX a bien évidemment pleinement bénéficié des avancées technologiques et scientifiques de ces dernières années. Ainsi, comme indiqué sur la figure 1, la technologie du laser de pompe est basé sur l'utilisation de cristaux de titane saphir. Le dispositif expérimental permet de disposer en sortie de pilote de plus de 2 joules à $10 \mathrm{~Hz}$. Cette énergie permet soit de réaliser des lasers XUV à haute cadence, auxquels par ailleurs on peut associer une source secondaire (IR, XUV) parfaitement synchronisée, soit après amplification de celle-ci dans un dernier étage de disposer de 40 Joules à une cadence de $0,1 \mathrm{~Hz}$.

This is an Open Access article distributed under the terms of the Creative Commons Attribution-Noncommercial License 3.0, which permits unrestricted use, distribution, and reproduction in any noncommercial medium, provided the original work is properly cited. 


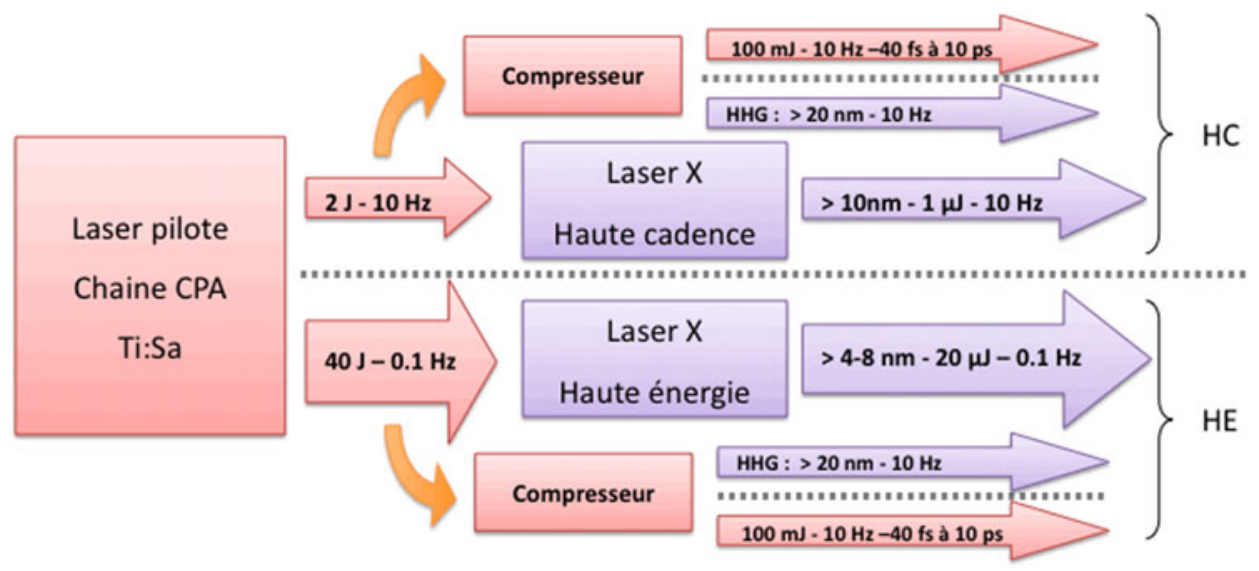

Figure 1. Représentation schématique du fonctionnement de LASERIX.

\section{LASERIX EST UNE RÉALITÉ : SOURCES LASERS XUV ET APPLICATIONS À UN TAUX DE RÉPÉTITION DE $10 \mathrm{HZ}$}

Durant la période (toujours en cours) de mise en service de la partie «haute énergie» de LASERIX, nous avons concentré nos recherches sur l'utilisation de l'énergie en sortie du pilote afin de disposer, dès le démarrage de l'installation de sources XUV exploitables pour des expériences d'irradiation, à un taux de répétition élevé de $10 \mathrm{~Hz}$.

\subsection{Développement du laser XUV émettant à $18.9 \mathrm{~nm}$}

L'essentiel de nos investigations à $10 \mathrm{~Hz}$ se concentre sur le laser XUV à ions molybdène nickéloïdes, émettant à $18.9 \mathrm{~nm}$.

La figure 2 illustre des résultats caractéristiques que nous avons obtenus quant à la production de cette source. Le schéma de pompage repose sur une configuration en incidence rasante (Grazing incidence pumping, GRIP) qui permet notamment de focaliser l'impulsion courte sous un angle rasant par rapport à la cible, afin d'optimiser le dépôt énergétique du pompage, dans des zones du plasma formé préalablement par l'impulsion longue - assurant la meilleure amplification possible [6-8].

\subsection{Optimisation de la source pour une expérience d'irradiation de plasmides d'ADN}

Forts des progrès évoqués précédemment, nous avons dans une seconde phase mené des travaux d'optimisation de la source pour une expérience d'application particulièrement sensible. En effet, il s'agit d'irradier des échantillons biologiques sous vide avec à la fois une grande précision et une extrême stabilité tir à tir (précision de l'ordre du milliradian). Cela suppose donc d'être capable de caractériser et de contrôler la source XUV en continu pendant plus de 20000 tirs.

Pour cela nous avons modifié l'arrangement expérimental afin de propager les deux impulsions (longue et courte) à partir de la même optique de focalisation, tout en permettant grâce à un dispositif optique supplémentaire introduit dans le pilote (de type Mach-Zehnder) de contrôler la variation de l'écart temporel et des rapports d'énergie entre les deux impulsions. Cette configuration de pompage, appelée «Double grazing incidence pumping» (DGRIP) [9,10], offre l'énorme avantage de s'affranchir des contraintes inhérentes à la superposition sur cible de 2 impulsions focalisées par des optiques différentes. Grâce à l'introduction de ce système et à différents travaux pour optimiser la source, nous 
Source Laser XUV
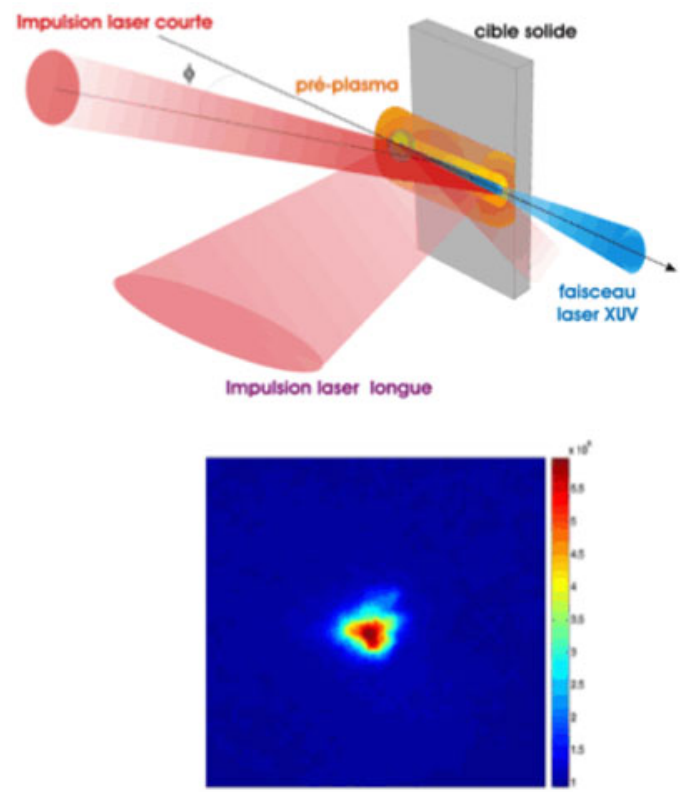

Source HHG XUV
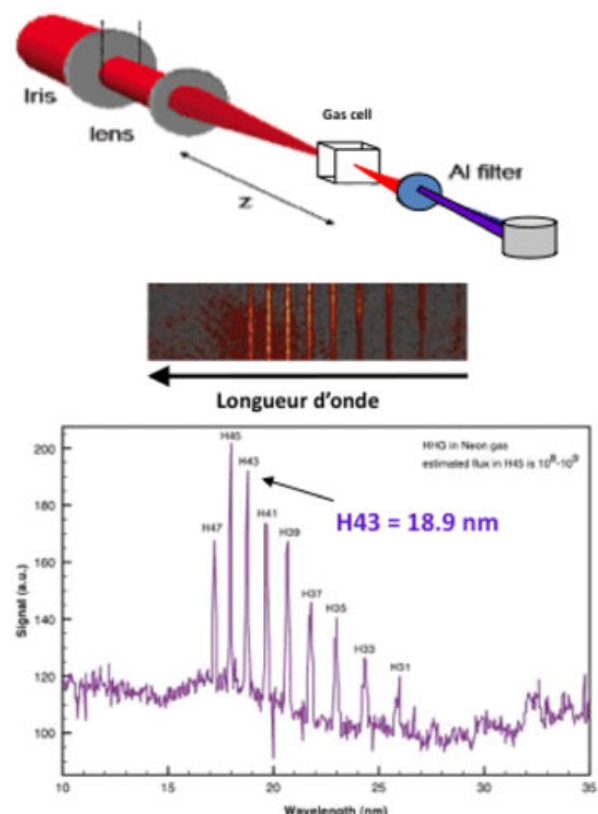

Figure 2. Représentation du schéma de pompage (images du haut) et images caractéristiques des sources obtenues à $18.9 \mathrm{~nm}$ (images du bas) dans le cas du laser XUV (image en champ proche en bas à gauche) et dans celui de la génération d'harmoniques obtenues à partir d'un gaz de Néon (spectre en transmission, en bas à droite).

sommes maintenant en mesure d'offrir une source parfaitement caractérisée et stable, à une cadence de tir de $10 \mathrm{~Hz}$, et ce en continu pendant une heure [11].

Ainsi, comme illustré en figure 3, nous avons clairement fait la démonstration de la production d'une source XUV à $18.9 \mathrm{~nm}$ avec une variation en énergie de $\pm 10 \%$ pendant plus de 20000 tirs, ainsi que de notre capacité à focaliser cette source sur un échantillon de la dimension du millimètre. Par ailleurs, cette expérience a permis de démontrer tout l'intérêt des sources laser XUV pour le domaine d'études des origines photoniques et/ou électroniques dans les simples et doubles cassures de plasmides d'ADN $[12,13]$.

\subsection{Perspectives d'accueil sur LASERIX à 10 Hz}

Cette phase d'investigations, menée dans des conditions par ailleurs non idéales (bâtiment préfabriqué suite au retard dans la construction de notre bâtiment sur le site de l'ENSTA), a permis de valider notre capacité à accueillir des utilisateurs extérieurs pour des expériences d'applications.

Le déménagement et la remise en service du laser, réalisés au premier semestre 2010, nous permet à ce jour d'accueillir nos premiers utilisateurs, tant pour des expériences d'irradiation que pour des expériences d'imagerie à partir de techniques interférométriques déjà démontrées $[14,15]$.

A partir de 2012, l'installation LASERIX offrira 4 semaines de temps de faisceaux au travers duconsortium européen LASERLAB-Europe. Par ailleurs, un comité national attribuera les temps d'expériences pour une période totale de 12 à 14 semaines. D'ores et déjà, les demandes de temps de faisceaux peuvent être déposées sur le site web de LASERIX (http: //www. laserix.u-psud.fr). L'équipe de LASERIX gardera un temps de faisceaux interne, de l'ordre de 16 à 20 semaines, pour à la fois mener à bien les investigations nécessaires au développement et à l'optimisation des sources 

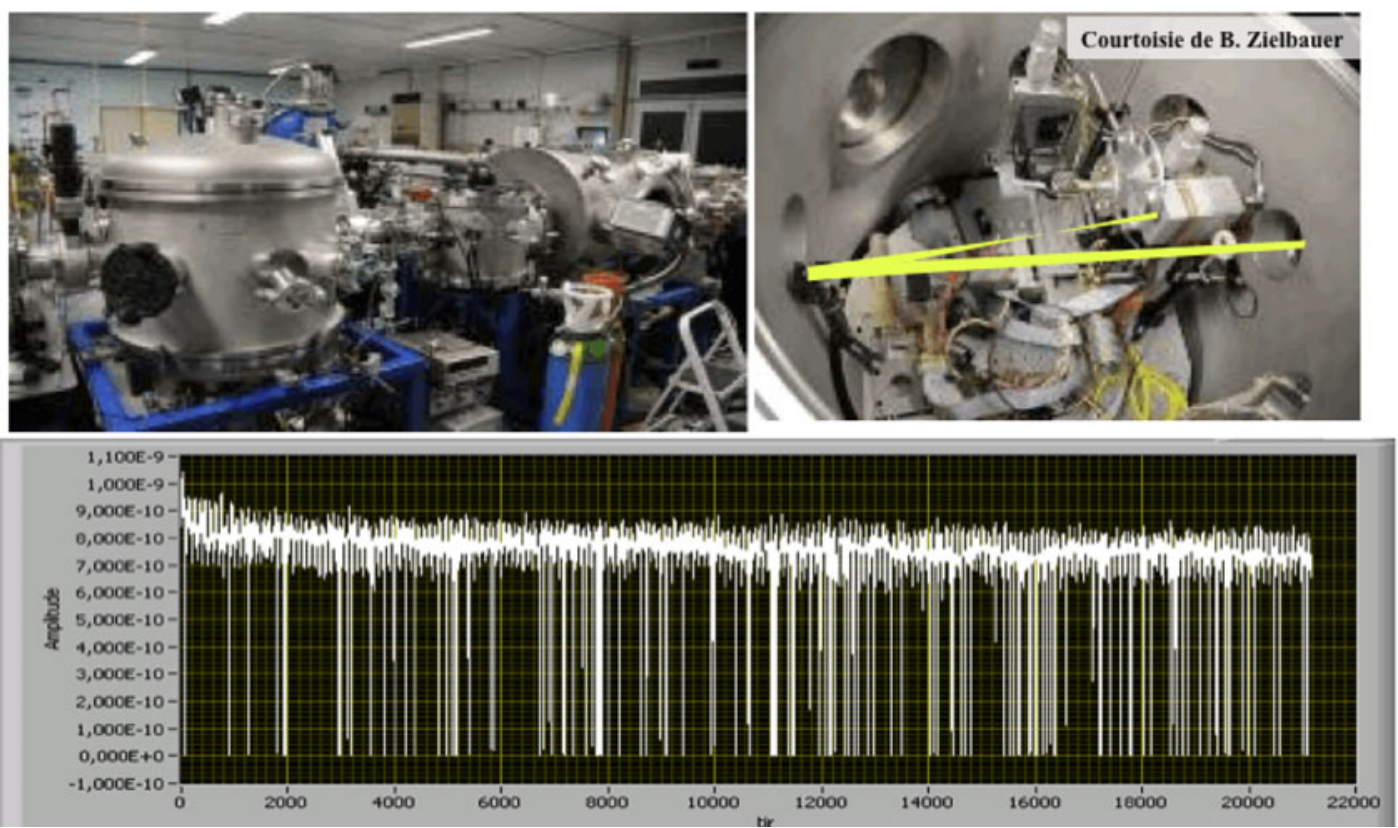

Figure 3. Photographies d'une expérience d'application de laser XUV (image en haut à gauche), de l'intérieur de l'enceinte (image en haut à droite), de l'enregistrement de la variation de l'énergie en fonction du nombre de tirs (image du bas).

XUV et favoriser des collaborations déjà existantes ou nouvelles, dans le cadre de l'intérêt scientifique de l'Université Paris-Sud.

\section{FUTURE UTILISATION DE LASERIX À PLEINE ÉNERGIE : 40 JOULES À 0,1 HZ}

En parallèle de ce travail d'investigations à $10 \mathrm{~Hz}$, nous mettons en place dans le nouveau bâtiment la partie de l'installation fournissant 40 Joules à un taux de répétition de $0,1 \mathrm{~Hz}$.

Ainsi, comme illustré sur la figure 4, LASERIX pourra à terme, et en parallèle (conformément au schéma de la figure 1) travailler à une cadence de $10 \mathrm{~Hz}$ (avec une énergie nominale de 2 Joules) et à $0,1 \mathrm{~Hz}$ (à une énergie avant compression estimée à 40 Joules).

Pour cela, l'année 2011 sera consacrée à l'obtention de 40 Joules, ainsi qu'à la démonstration de la compression de 20 Joules dans l'enceinte «FERRARIX» dédiée à la production de sources laser XUV à haute énergie. Les premières investigations reposeront sur nos travaux en configuration de pompage DGRIP. Les premiers photons XUV devraient se propager depuis cette enceinte à la fin de l'année 2011, et des premières expériences seront réalisées sur le temps interne au cours du premier semestre 2012, avant une ouverture à la communauté prévue au second semestre 2012.

Au delà de la possibilité de développer des laser XUV à plus courte longueur d'onde (inférieures à $7 \mathrm{~nm}$ ), le principal intérêt résidera dans la capacité de réaliser des expériences de type pompe-sonde, compte tenu de la parfaite synchronisation des faisceaux distribués.

\section{CONCLUSION ET PERSPECTIVES}

Ainsi, la facilité LASERIX est maintenant opérationnelle en continu à une cadence de tirs de $10 \mathrm{~Hz}$. Nous maîtrisons le développement de sources laser XUV (entre 10 et $32 \mathrm{~nm}$ ), et avons fait la démonstration de notre capacité à les caractériser et surtout à les stabiliser pour des expériences 


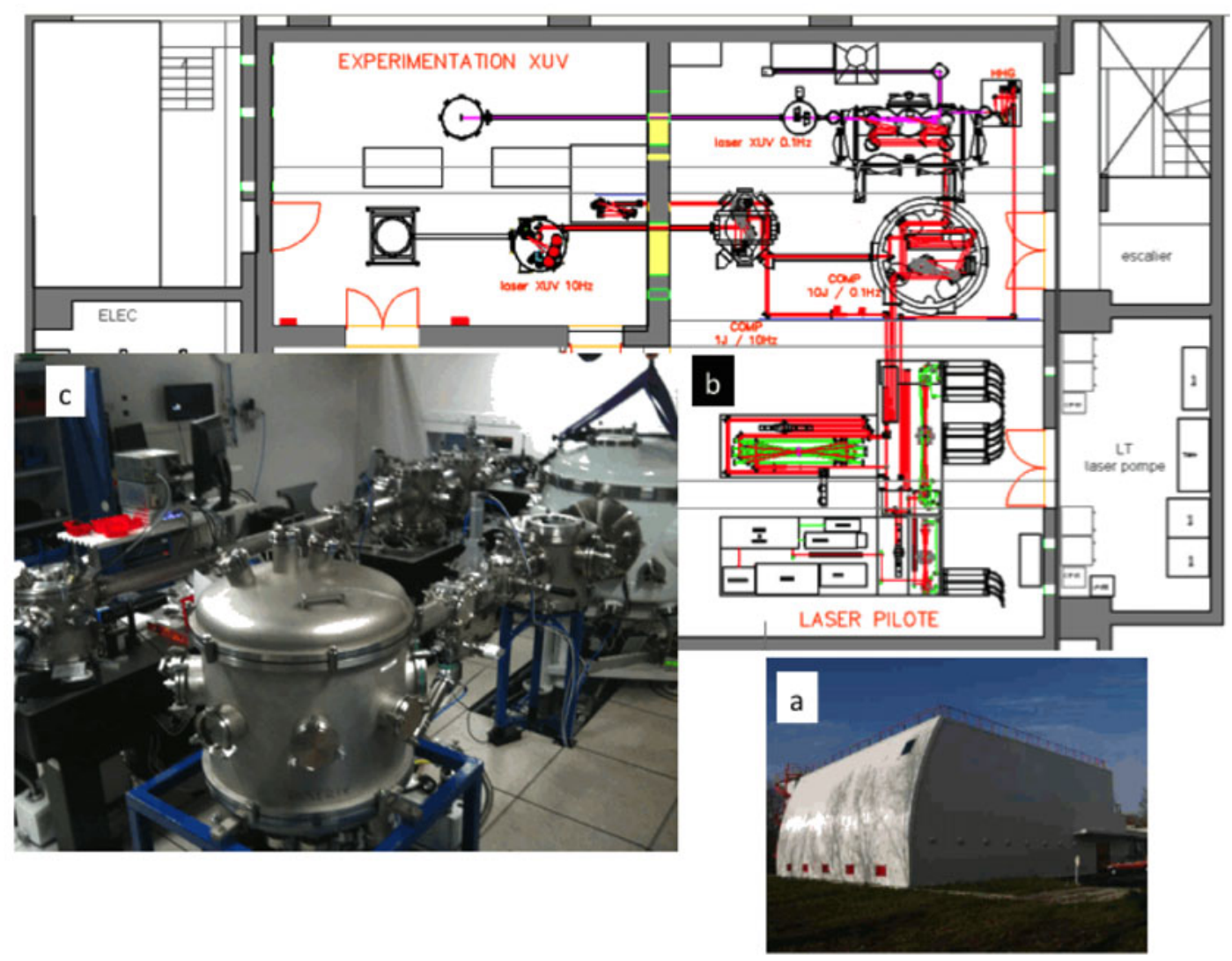

Figure 4. Photographies du «nouveau» bâtiment accueillant l'installation LASERIX depuis 2010 (a), de la répartition expérimentale de LASERIX dans 2 salles du sous-sol du bâtiment (b) et de l'arrangement expérimental de la salle «utilisateurs» (c).

d'application nécessitant une haute cadence. LASERIX sera ouvert aux utilisateurs extérieurs à partir du mois de janvier 2011 pour la partie «2 Joules» à un taux de répétition de $10 \mathrm{~Hz}$. Le premier semestre verra par ailleurs le déploiement de la partie «40 Joules» à un taux de répétition de $0.1 \mathrm{~Hz}$. Le second semestre 2011 sera consacré alors à des développements en interne par l'équipe LASERIX, précédant l'accueil des utilisateurs à l'horizon de 2012.

\section{Remerciements}

La majeure partie des progrès réalisés quant aux sources XUV et leur utilisation pour les applications repose sur l'utilisation d'optiques (de transport et de focalisation) de grande qualité dans le domaine des rayons XUV. Elles sont d'une grande utilité autant pour le conditionnement du faisceau lui-même que pour la prise en compte des besoins spécifiques des différentes applications (imagerie ou interférométrie). L'essentiel de nos optiques, aboutissant à des résultats scientifiques de première importance, sont le fruit d'un travail et d'une expertise internationalement reconnus du laboratoire Charles Fabry de l'Institut d'Optique «Graduate School». Nous les remercions chaleureusement pour leur compétence et leur investissement de qualité dans nos collaborations scientifiques.

\section{Références}

[1] A. Klisnick et al., JOSA B 17,1093-1097 (2000). 
[2] D. Ros et al, Laser and Particle Beams Journal 20, 23 (2002).

[3] A. Klisnick et al., J.Q.S.R.T. 99 Issues 1-3, 370-380 (2006).

[4] S. Kazamias et al., J. Phys. IV France 138 13-19 (2006).

[5] O. Guilbaud et al., Proc. of UVX 2008 - EDP Science 57-63 (2009).

[6] R. Keenan et al., Phys. Rev. lett. 94, 103901 (2005).

[7] K. Cassou et al., Opt. Lett. 32 139-141 (2006).

[8] S. Kazamias et al., Phys. Rev. A 77 (2008).

[9] D. Zimmer et al., Opt. Express 1610398 (2008).

[10] D. Zimmer et al., Opt. Lett. 35450 (2010).

[11] B. Zielbauer et al., Appl. Phys. B - lasers and Optics - 100731 (2010).

[12] K. Cassou et al., J. Phys. IV 127, 177-180 (2005).

[13] S. Lacombe et al., Soft X-Ray lasers and Applications VIII 7451 (2009).

[14] P. Zeitoun et al., Phys. Rev. B 60 11089-11094 (1999).

[15] H. Tang et al., Appl. Physics B 78 975-977, (2004). 